\title{
Mode of Clinical Presentation and Delayed Diagnosis of Turner Syndrome
}

\author{
Louise J Apperley¹, Urmi Das², Renuka Ramakrishnan², Poonam Dharmaraj², Jo Blair², Mohammed Didi², Senthil \\ Senniappan ${ }^{2}$ \\ Authors declare no conflict of interest
}

${ }^{1}$ Mid Cheshire Hospitals NHS Foundation Trust, Leighton, UK $\quad$ 2. Alder Hey Children's NHS Foundation Trust, Liverpool, UK.

\section{Background}

Early diagnosis of girls with Turner syndrome (TS) is essential to provide timely intervention and support. Based on the findings by Savendahl and Davenport ${ }^{1}$, the proposed guidelines for screening girls for TS were used (Table1).

\section{Table 1: Guidelines for screening for TS 1}

Any girl with one or more of the following*:

1. Unexplained short stature (height $<5^{\text {th }}$ percentile)

2. Webbed neck

3. Peripheral lymphoedema

4. Coarctation of the aorta

5. Delayed puberty

OR

Any girl who has at least two or more of the following:

1. Nail dysplasia

2. High arched palate

3. Short $4^{\text {th }}$ metacarpal

4. Strabismus

* Other suggestive features include: non-verbal learning disability, epicanthial folds, ptosis, cubitus vagus, multiple naevi, renal malformations, bicuspid $\mathrm{AV}$, recurrent $\mathrm{OM}$ and need for glasses

\section{Aim}

The aim of the study was to determine the age and clinical features at the time of presentation to identify potential delays in diagnosis of TS.

\section{Method}

Retrospective data collection on age at diagnosis, reason for karyotype analysis and presenting clinical features was collected from the medical records of 67 girls with TS.

30

25

20

تِّ

15

10

5

0

Lymphoedema

Dysmorphic

Amb genitalia

Features

FTT
60

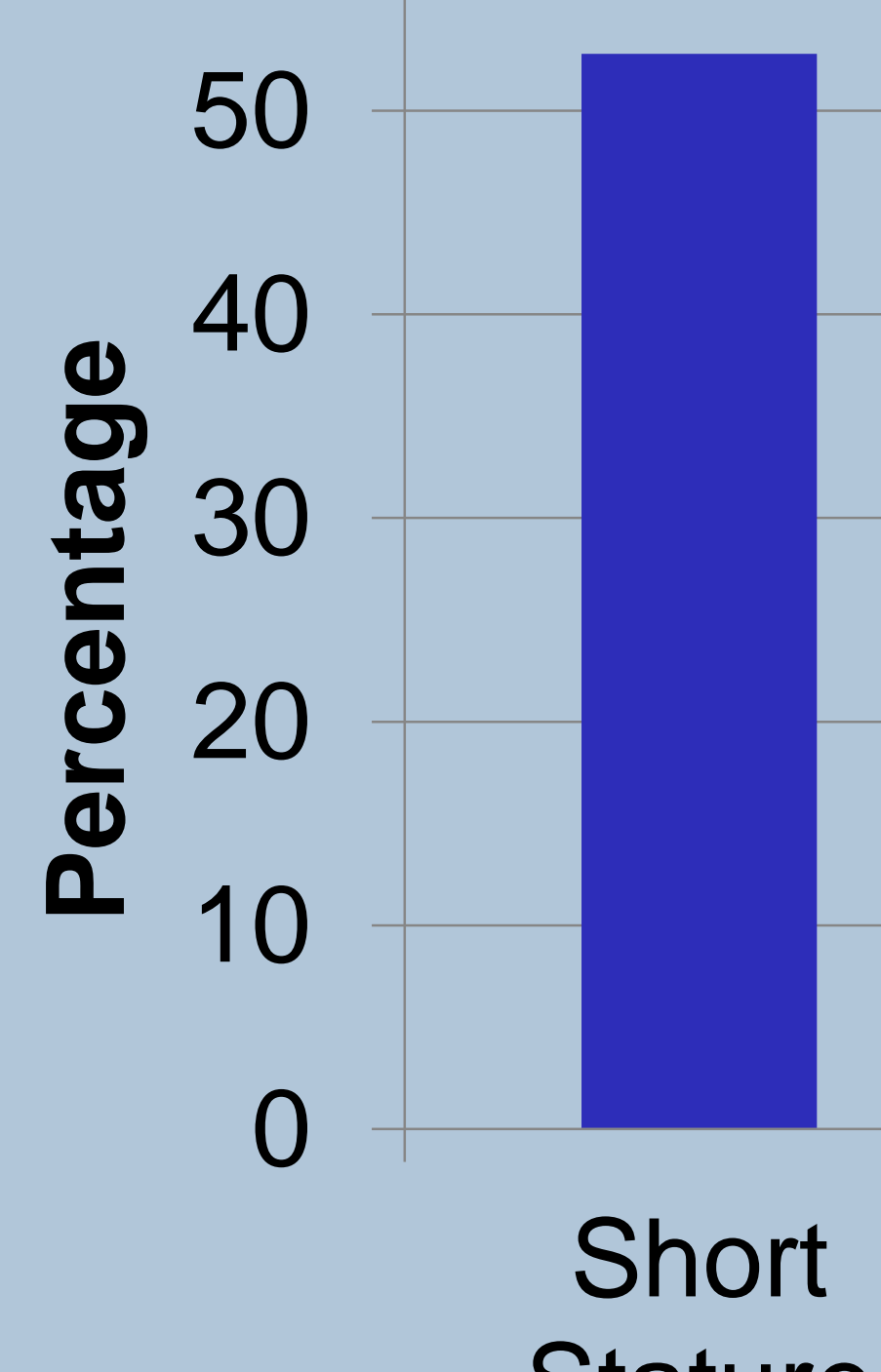

Short

Inadequate Dysmorphic Seizures

Cardiac Incidental

Reason for Screening

Figure 2: Initial clinical presentation for patients diagnosed in their childhood years (aged 1-12 years)

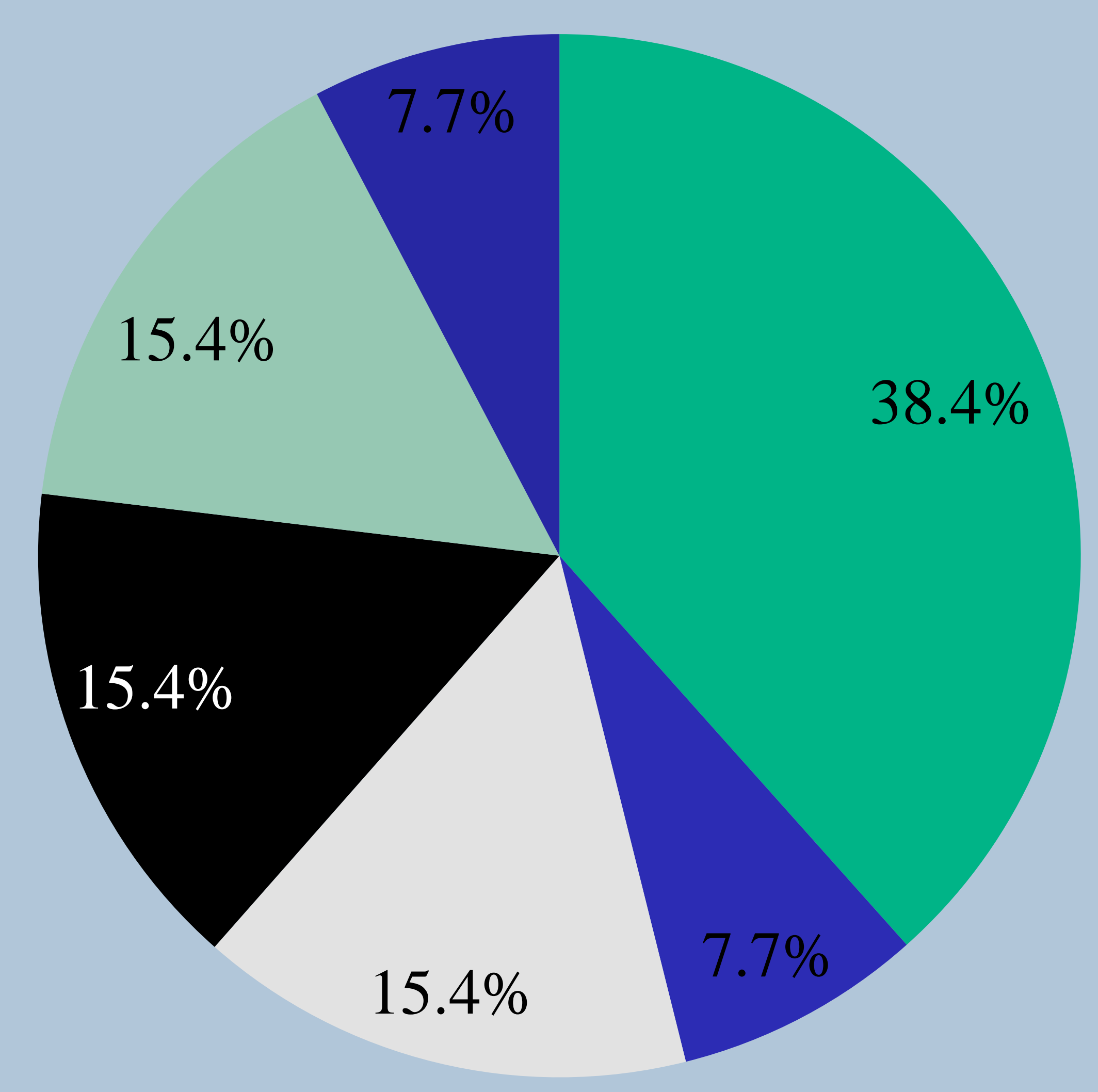

Short Stature (SS)

- SS and Delayed

Puberty

SS and Amenorrhoea

- SS and Irregular

periods

Delayed Puberty

- Inadequate

Information

Figure 3: Initial clinical presentation for patients diagnosed in their adolescent years (aged 12-18 years)

\section{Results}

- Mean age of diagnosis was $5.89( \pm 5.3)$ years, ranging from prenatal to 17.9 years.

- Overall only $42 \%$ of the girls were diagnosed before the age of 5 years.

- $10 \%$ were diagnosed antenatally ( $29 \%$ secondary to maternal age).

- $16 \%$ of the patients were diagnosed in infancy (Figure 1).

- $54 \%$ were diagnosed in childhood (Figure 2). 6 of the 36 children should have been screened at birth if using the screening guidelines.

- Finally, 20\% were diagnosed between the ages of 12 and 18 years old (Figure 3 ). 2 of these adolescents should have been screened at birth if using the proposed guidelines.

- At least $12 \%$ of girls fulfilled the criteria for earlier screening but were diagnosed only at a later age (mean age $=8.78$ years).

- The actual duration of delay in children presenting with short stature could not be ascertained due to lack of height measurements prior to seeking specialist opinion.

\section{Conclusions}

- The majority of girls with TS were diagnosed only after the age of 5 years.

- Short stature triggered evaluation for most patients diagnosed in childhood and adolescence.

- Lack of community height-screening programme and lack of awareness could have led to potential delays in diagnosing TS.

- New strategies for earlier detection of TS are needed. 